\title{
Response to anti-PD1 therapy with nivolumab in metastatic sarcomas
}

\author{
L. Paoluzzi ${ }^{*} \mathbb{D}$, A. Cacavio ${ }^{1}$, M. Ghesani², A. Karambelkar², A. Rapkiewicz³ , J. Weber ${ }^{1}$ and G. Rosen ${ }^{1}$
}

\begin{abstract}
Background: Manipulation of immune checkpoints such as CTLA4 or PD-1 with targeted antibodies has recently emerged as an effective anticancer strategy in multiple malignancies. Sarcomas are a heterogeneous group of diseases in need of more effective treatments. Different subtypes of soft tissue and bone sarcomas have been shown to express PD-1 ligand.

Methods: We retrospectively analyzed a cohort of patients (pts) with relapsed metastatic/unresectable sarcomas, who were treated with nivolumab provided under a patient assistance program from the manufacturer. Pts underwent CT or PET/CT imaging at baseline and after at least four doses of nivolumab; RECIST 1.1 criteria were used for response assessment.

Results: Twenty-eight pts with soft tissue (STS, $N=24)$ or bone sarcoma $(N=4)$, received IV nivolumab $3 \mathrm{mg} /$ kg every 2 weeks from July 2015. Median age was 57 (24-78), male:female ratio was 14:14; the median number of nivolumab cycles was eight. Eighteen pts concomitantly received pazopanib at 400-800 mg daily. The most common side effect was grade 1-2 LFT elevations; grade 3-4 toxicity occurred in five patients (colitis, LFT elevations, pneumonitis). Twenty-four pts received at least four cycles. We observed three partial responses: one dedifferentiated chondrosarcoma, one epithelioid sarcoma and one maxillary osteosarcoma (last two patients on pazopanib); nine patients had stable disease including three leiomyosarcomas; 12 patients had progression of disease including 4 leiomyosarcoma. Clinical benefit (response + stability) was observed in $50 \%$ of the evaluable patients.
\end{abstract}

Conclusions: These data provide a rationale for further exploring the efficacy of nivolumab and other checkpoint inhibitors in soft tissue and bone sarcoma.

Keywords: Sarcoma, Immunotherapy, Nivolumab, PD-1, Check point inhibitors

\section{Background}

Soft tissue (STS) and bone sarcomas are a heterogeneous group of diseases with an estimated 15,000 new cases in the US in 2016, and more than 50 different subtypes [1, 2]. Given their rarity and diversity, enrollment into prospective clinical trials has been very challenging, even in the context of cooperative groups. Despite new studies elucidating the genomic basis and the sensitivity to chemotherapy of specific subtypes, the overall prognosis of patients with metastatic sarcoma remains poor in most cases. Since 2012, new drugs such as pazopanib,

\footnotetext{
*Correspondence: luca.paoluzzi@nyumc.org

1 Department of Medicine, NYU Langone Medical Center, New York, NY, USA

Full list of author information is available at the end of the article
}

and for more specific subtypes, trabectedin and eribulin, have been approved for patients who have relapsed after front line chemotherapy, but the response rates for these agents remains suboptimal [3]. Immunotherapy has recently provoked great interest in oncology after phase III clinical trials have shown significant efficacy in chemotherapy-resistant malignancies such as metastatic melanoma or renal cell carcinoma, and activity in chemotherapy-sensitive histologies including non-small cell lung cancer, head and neck cancer, MSI-high colon cancer and Hodgkin's lymphoma.

Programmed death 1 (PD-1) is a surface receptor expressed on activated and exhausted $\mathrm{T}$ cells, which mediates inhibition of activation, cytokine secretion and lytic activity upon binding with its ligands (PD-L1 and 
PD-L2). The role of the PD-1/PDL-1 axis in suppression of $\mathrm{T}$ cell activation and its targeting through specific monoclonal antibodies, has been the basis for the success achieved in a number of clinical trials [4]. Tumor PD-L1 expression has been reported in up to $65 \%$ of different subtypes of sarcomas [5]. The degree of PD-1 positivity in tumor-infiltrating lymphocytes (TILs) and PD-L1 expression in tumor specimens from 105 cases of soft tissue sarcomas, has been correlated with a poorer prognosis and more aggressive disease [6]. While preclinical studies and retrospective analyses of clinical data may provide a rationale for immune-mediated strategies against sarcoma, there are currently very limited clinical data to support the use of anti-PD-1 antibodies in this setting. We report herein a retrospective series of twentyeight patients with metastatic or locally advanced soft tissue or bone sarcoma who received the PD-1 antibody nivolumab under a patient assistant program off protocol, with or without pazopanib. We describe for the first time clinical benefit in different subtypes, as shown by disease regression or stabilization.

\section{Patients and methods}

Between July 2015 and August 2016, twenty-eight patients with a diagnosis of soft tissue or bone sarcoma were treated with nivolumab. All patients but two, previously received one line of systemic treatment; patients receiving pazopanib before starting nivolumab were continued on this treatment given concern for disease flare after discontinuation, as described for other tyrosine kinase inhibitors [7]. The following data were recorded for all patients: gender, age, location of the primary sarcoma, stage, median number of prior therapies, ECOG performance status, number of cycles of nivolumab administered. Nivolumab was given at the standard dose of $3 \mathrm{mg} / \mathrm{kg}$ IV every 2 weeks; the drug was provided by the manufacturer under a patient assistant program. Complete blood count, electrolytes, liver and kidney function tests were performed before each cycle of treatment, more often if clinically indicated; all toxicities were recorded at each visit (at least every 2 weeks), as per NCI CTCAE v.4.0. Baseline scans consisted of PET/CT or CT scans with IVC, imaging was repeated every two or three months. Next generation sequencing to determine the presence of specific mutations in a panel of 50 genes was performed in one patient with a dedifferentiated chondrosarcoma responding to nivolumab alone; the polymerase chain reaction (PCR) product for specific mutations was sequenced on an Ion Torrent PGM instrument (Thermo Fisher Scientific, Waltham, MA). PD-L1 expression was assessed in selected patients who had evaluable tissue for testing $(\mathrm{N}=10)$ and performed at Esoterix Genetic Laboratories (Integrated Oncology,
New York, NY). PD-L1 positive was defined as a tumor proportion score (\% of at least 100 viable tumor cells with complete or partial $1+$ membrane staining) of $50 \%$ for at least 100 viable tumor cells exhibiting membrane staining. For PD-L1 detection, we used the PD-L1 IHC 28-8 pharmDx assay (Dako North America Inc, CA, USA).

\section{Results}

\section{Patients and treatment}

The clinical characteristics of the patients are shown in Table 1. A total of 28 patients with a diagnosis of metastatic $(n=26)$ or unresectable $(n=2)$ soft tissue or bone sarcoma received IV nivolumab every 2 weeks; median age was 57 years, female to male ratio was 14:14, ECOG performance status was $0-1$ for 24 patients, and 2 for the remaining 4 patients. Eighteen patients received concomitant pazopanib. The median number of prior systemic

\section{Table 1 Patient baseline characteristics}

\begin{tabular}{|c|c|}
\hline Factor & No \\
\hline \multicolumn{2}{|l|}{ Age } \\
\hline Median & 57 \\
\hline Range & $24-78$ \\
\hline \multicolumn{2}{|l|}{ Sex } \\
\hline Female & 14 \\
\hline Male & 14 \\
\hline Soft tissue sarcoma & 24 \\
\hline Bone sarcoma & 4 \\
\hline \multicolumn{2}{|l|}{ Location } \\
\hline Extremity & 6 \\
\hline Abdomen/pelvis & 12 \\
\hline Axial & 2 \\
\hline Head/neck & 5 \\
\hline Chest & 3 \\
\hline \multicolumn{2}{|l|}{ Stage } \\
\hline IV & 26 \\
\hline Unresectable & 2 \\
\hline \multicolumn{2}{|l|}{ ECOG PS } \\
\hline $0-1$ & 24 \\
\hline 2 & 4 \\
\hline \multicolumn{2}{|l|}{ Nivolumab } \\
\hline Cycles (median) & 8 \\
\hline Range & $1-26$ \\
\hline On Pazopanib & 18 \\
\hline \multicolumn{2}{|c|}{ Prior treatments (including neoadjuvant/adjuvant) } \\
\hline Median & 2 \\
\hline Range & $0-6$ \\
\hline Anthracycline & 15 \\
\hline Ifosfamide & 10 \\
\hline Gemcitabine & 9 \\
\hline Docetaxel & 7 \\
\hline
\end{tabular}


treatments, including neoadjuvant and adjuvant chemotherapy was 2 (range 0-6). Sarcoma subtypes are listed in Additional file 1: Table S1; twenty-four patients had a diagnosis of a soft tissue sarcoma with leiomyosarcoma (LMS) being the most common subtype $(n=7)$; four patients had conventional osteosarcoma (OS).

\section{Safety}

Table 2 shows all the adverse events (AE); most side effects were grade 1-2 with a predominance of LFT abnormalities (8 out of 10 patients on pazopanib). Grade 3-4 AE were experienced by four patients, all on pazopanib. One patient experienced grade 3 elevation of AST/ALT/alkaline phosphatase and grade 4 bilirubin elevation after two cycles of nivolumab; liver biopsy was consistent with drug related hepatitis; she discontinued treatment with normalization of bilirubin, and improvement of ALT/AST to grade 1. A second patient experienced grade 3 ALT elevation that improved to grade 1 once both nivolumab and pazopanib were discontinued and high dose steroids (prednisone $1 \mathrm{mg} / \mathrm{kg} /$ daily) were administered with a slow taper over about 2 months; she subsequently restarted treatment with both drugs. A third patient had grade 4 AST/grade 3 ALT/alkaline phosphatase elevations with grade 3 pneumonitis that required intubation; he recovered after high dose steroids and he was able to restart pazopanib only, after LFTs normalized. A fourth patient experienced grade 3 colitis that significantly improved with high dose steroids; she was restarted on treatment with both pazopanib and nivolumab until she progressed.

\section{Efficacy outcomes}

Twenty-four patients were evaluable for response (Fig. 1). Four patients were not evaluable for the following reasons: liver toxicity after 2 cycles $(\mathrm{n}=1)$; patients lost at follow up $(\mathrm{n}=2)$, concomitant radiation therapy $(\mathrm{n}=1)$. We observed three partial responses (PR) and they included: a 74 year-old patient with a dedifferentiated chondrosarcoma (DC), after six cycles of nivolumab alone with PR maintained after 26 cycles (Fig. 2a; Additional file 1: Figure S1). The NGS-tumor 50 panel only showed non-synonymous variants of unknown significance for the PIK3CA and TP53 genes; PD-L1 staining was $20 \%$. A second PR was observed in a 46 year-old female with a relapsed, OS of the left maxilla. She had a minimal clinical response to nivolumab given for four cycles; pazopanib was then added and it was given for only one month (Fig. 2b). The rationale to add pazopanib after 4 cycles of nivolumab alone, relied on the following considerations: (1) the original lesion showed abundant vascularization; (2) pazopanib targets the vascular endothelial growth receptors VEGF-1, VEGFR-2, VEGF-3; (3) nivolumab alone was tolerated very well; (4) a resection of this challenging lesion could potentially give the best chance for a long progression free survival in this young patient. After one month of pazopanib, her facial lesion significantly regressed and the patient had major clinical benefit in terms of improved eating habits and pain control. At that point we thought it was in the patient's best interest to undergo surgery. At the time of resection, the tumor showed extensive necrosis and margins were negative. PD-L1 in this patient was $<5 \%$. A third PR was observed in a 24 year-old man with a proximal type epithelioid sarcoma (EpS) metastatic to the lung progressing on pazopanib. We decided to continue pazopanib given the concern for disease flare after discontinuation as described for other tyrosine kinase inhibitors [7]. This patient had a PR after four cycles of nivolumab, progression $(\mathrm{PD})$ due to a new lesion in the left lung after

Table 2 Safety

\begin{tabular}{|c|c|c|c|c|c|c|}
\hline & \multicolumn{3}{|l|}{ Grade 1-2 } & \multicolumn{3}{|l|}{ Grade 3-4 } \\
\hline & On Pz (No.) & NO Pz (No.) & Total (No.) & On Pz (No.) & NO Pz (No.) & Total (No.) \\
\hline \multicolumn{7}{|l|}{ Hematologic } \\
\hline Anemia & 3 & 1 & 4 & - & - & - \\
\hline Neutropenia & 1 & - & 1 & - & - & - \\
\hline Thrombocytopenia & 1 & 1 & 2 & - & - & - \\
\hline \multicolumn{7}{|l|}{ Non-hematologic } \\
\hline Diarrhea & 3 & & 3 & 1 & & 1 \\
\hline Pneumonitis & 1 & & 1 & 1 & & 1 \\
\hline Rash & 3 & 1 & 4 & - & - & - \\
\hline Hypothyroidism & 6 & 2 & 8 & - & - & - \\
\hline LFTs & 8 & 2 & 10 & 3 & & 3 \\
\hline
\end{tabular}

Toxicity was graded as per NCI CTCAE v4.0

Pz pazopanib, $400-800 \mathrm{mg}$ po daily; LFTs liver function tests abnormalities 


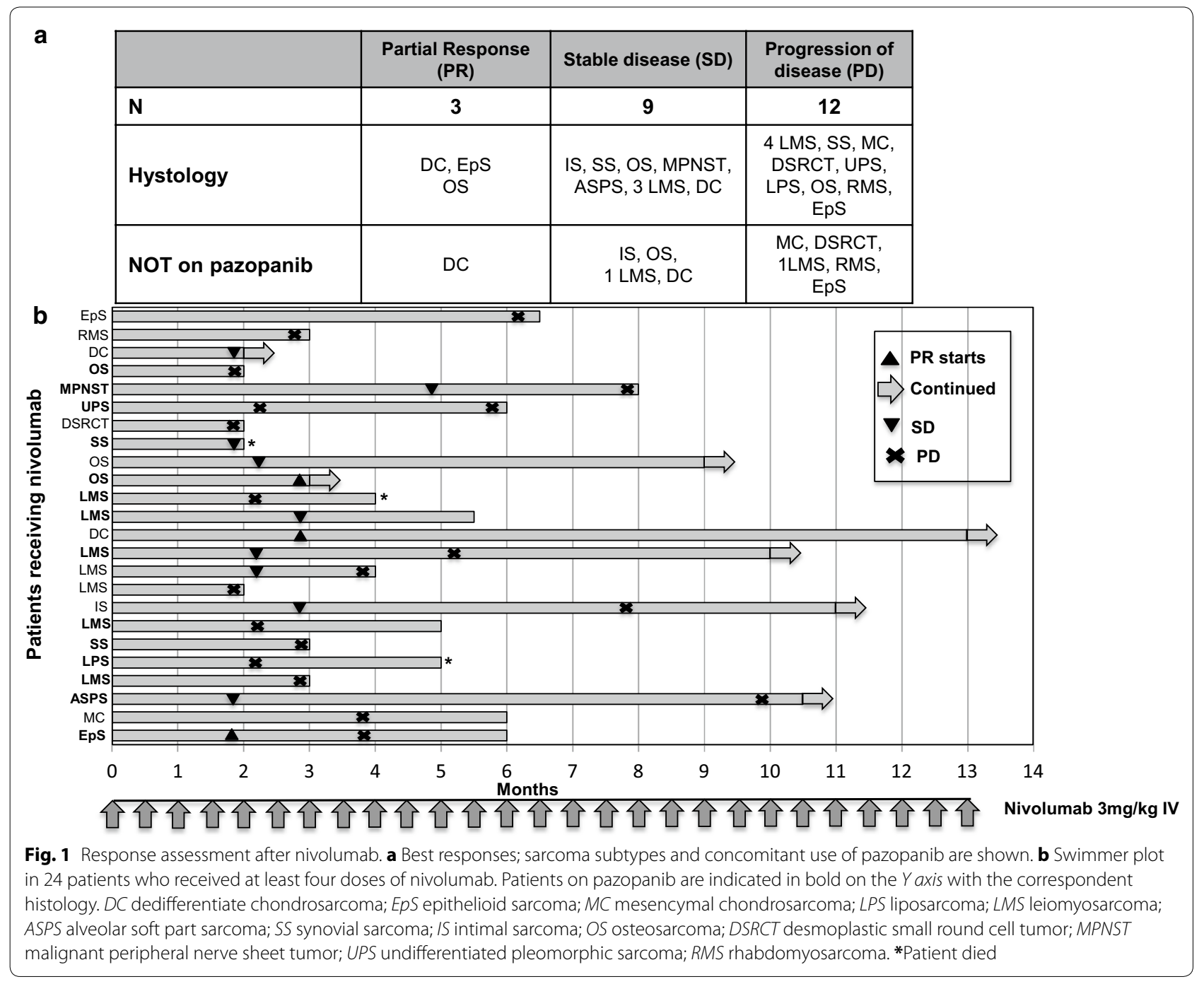

four additional cycles; he had further PD in the lung after four more cycles and nivolumab was stopped.

Nine patients had stable disease (SD): five patients received pazopanib plus nivolumab while four patients received nivolumab alone (one dedifferentiated chondrosarcoma, one leiomyosarcoma, one intimal sarcoma and one osteosarcoma).

A patient with an alveolar soft part sarcoma (ASPS) progressing on pazopanib, with a slight PD ( 25\% increase per RECIST criteria) after nine cycles of nivolumab, he is overall asymptomatic and continues treatment with both drugs; two patients with uterine LMS, both progressing on pazopanib alone, one had $\mathrm{SD}$ after five cycles (PD after 6 more) and the other SD after six cycles (nivolumab stopped after five more cycles because of pneumonitis); a patient with a LMS of the vulva had SD after five cycles of nivolumab alone but a
PD after three additional cycles; a patient with an intimal sarcoma (IS) of the right pulmonary artery metastatic to both lungs, experienced a SD after six cycles of nivolumab alone (the primary site was not evaluable because previously irradiated); he had PD after six additional cycles but continues nivolumab because he is asymptomatic; a patient with a synovial sarcoma (SS) had a SD after four cycles, but died from complications during surgery for repair of a pulmonary artery pseudo-aneurysm; a patient with a maxillary OS had SD after five cycles of nivolumab alone (confirmed after a total of 16 cycles); a patient with a malignant peripheral sheet tumor (MPNST), on pazopanib, had stability after four cycles but progressed after six additional cycles of nivolumab; a patient with a dedifferentiated myxoid chondrosarcoma (MC) had SD after four cycles of nivolumab. 


\section{a}

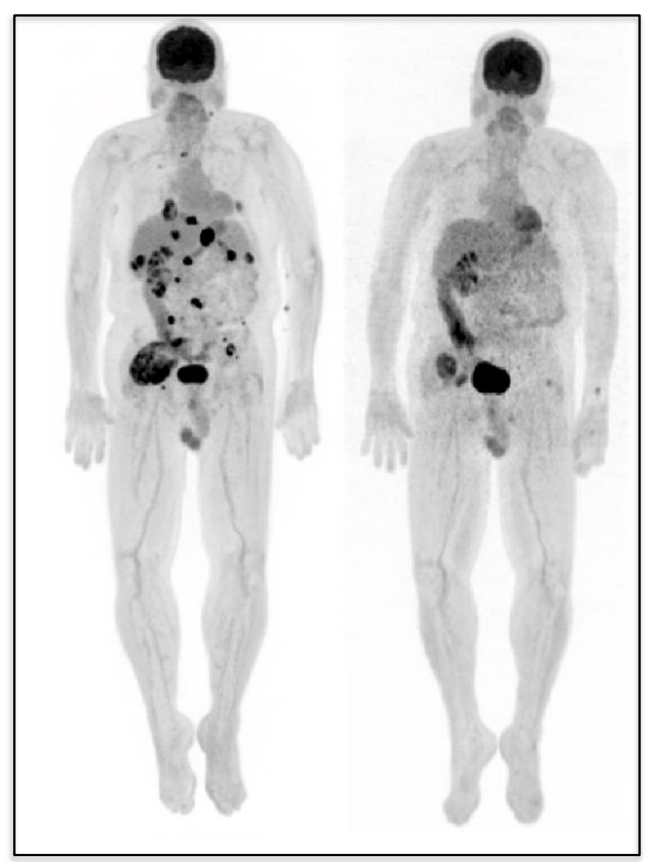

b

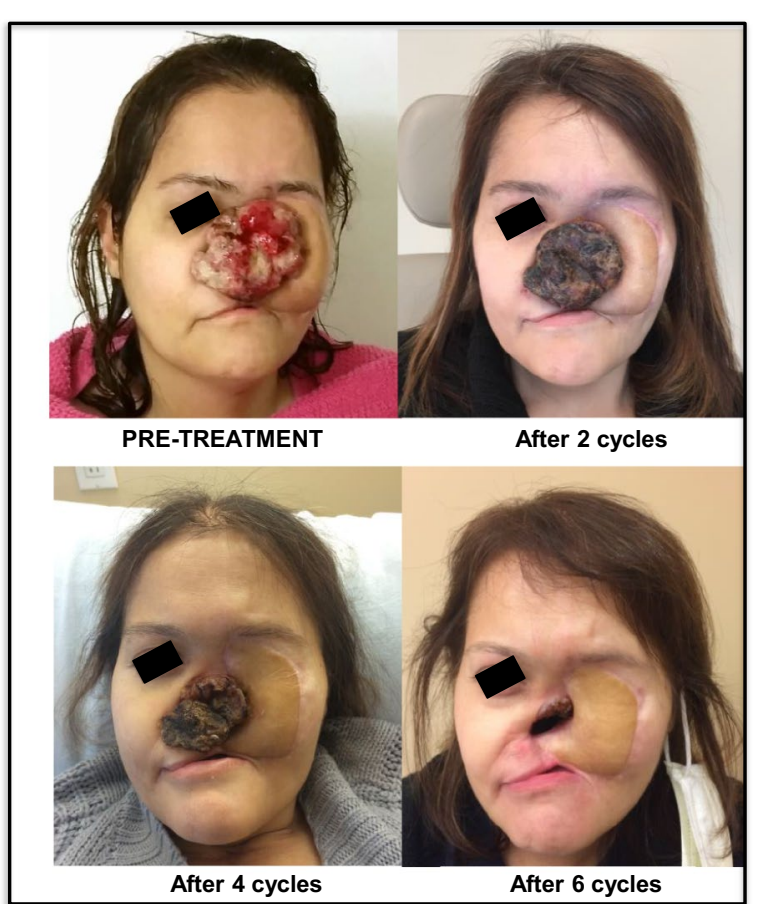

Fig. 2 Partial response (PR) to nivolumab in 2 patients. a PET/CT of a 74 year-old male with metastatic dedifferentiated chondrosarcoma after six cycles of nivolumab alone; he is maintaining a PR after 26 cycles. b 46 years-old woman with osteosarcoma, treated with nivolumab for six cycles; pazopanib 800 mg p.o. daily was started after 4 cycles of nivolumab. She underwent resection with negative margins

Overall, 12 out of 24 evaluable patients had clinical benefit $(\mathrm{PR}+\mathrm{SD})$. Twelve patients had PD: four patients with LMS (three on pazopanib): one transferred care to another hospital after six cycles, one had PD after five cycles, confirmed after five more, and switched to another treatment, one received five cycles and died after an accidental fall, a last one had a PD after four cycles on nivolumab alone. Additional PD in patients on pazopanib included: one with a MC after four cycles (confirmed after 4 more), one with a liposarcoma (LPS) after five cycles (confirmed after 6 more), one with an OS after four cycles; a patient with a SS after six cycles complicated by severe pneumonitis; one patient with an undifferentiated pleomorphic sarcoma (UPS) of the right upper extremity after five cycles (confirmed after 7 more). Patient with PD on nivolumab alone included: one with a desmoplastic small round cell tumor (DSRCT) after four cycles; one patient with a rhabdomyosarcoma (RMS) after six cycles; one with an EpS after 13 cycles.

\section{Discussion}

Patients with metastatic soft tissue sarcomas generally have a poor prognosis, with low response rates after first line chemotherapy [3]. Of note, the tyrosine kinase inhibitor pazopanib was approved by the FDA in 2012 on the basis of a phase III randomized trial showing improved
PFS in the second line setting; the overall response rate was only $6 \%$ [8].

Multiple recent genomic studies have provided better insight into sarcoma biology through a more accurate classification by molecular subtype, identification of recurrent mutations in oncogenic pathways and evidence of epigenetic dysregulation [9]. Barretina et al. [10] for example, recently provided a comprehensive database of sarcoma genome alterations in 207 samples of STS; despite their elucidation of genes and signaling pathways not previously associated with STS, we still lack appropriate pharmacologic tools for targeting specific genomic alterations.

Several subtypes of STS are characterized by specific chromosomal translocations which result in unique fusion proteins; while many of them function as transcription factors, making their therapeutic targeting quite challenging, these proteins may represent attractive targets from an immunotherapy standpoint [11]. Immunogenicity of sarcoma is supported by several preclinical studies and some clinical data with human sarcoma specimens.

Immunotherapeutic strategies in sarcoma have included cytokine-based immunotherapies, treatment with muramyl tripeptide phosphatidyl ethanolamine in osteosarcoma, vaccines and adoptive immunotherapy to 
cite a few examples, although none have appeared promising to date [12]. Our retrospective analysis shows the potential clinical benefit from treatment of soft tissue and bone sarcomas with the anti-PD1 antibody nivolumab.

This is not a prospective study, and given the retrospective nature of this series, it has several limitations; data on patients who received either nivolumab alone $(\mathrm{N}=10)$ or nivolumab + pazopanib $(\mathrm{N}=18)$, were pulled together in order to capture a possible signal of activity from immunotherapy (alone or in combination) that may be helpful for a following prospective study. Additionally, this is small study with multiple hystologies included: the largest group of patient had a diagnosis of leiomyosarcoma $(\mathrm{N}=7)$, but most subtypes are represented by only 1 or 2 patients.

In our series we showed disease improvement or stabilization in 12/24 patients evaluable for response. Eighteen out of twenty-eight patients concomitantly received pazopanib, however 1 partial response was observed in a dedifferentiated chondrosarcoma on nivolumab alone. Another response was seen in a patient with an unresectable maxillary OS who received four cycles of nivolumab and only one month of pazopanib that was started after the 4 cycles of nivolumab. A third patient with an epithelioid sarcoma, progressing on pazopanib, had a partial response after only four cycles of nivolumab; unfortunately he progressed after four additional cycles. Of note, overall responses were observed in some subtypes that are generally resistant to traditional chemotherapy such as dedifferentiated chondrosarcoma and epithelioid sarcoma. Interestingly, all the three aforementioned patients received adjuvant radiation therapy up to 20 years before, bringing up the possibility of a distant abscopal effect as hypothesized for other diseases such as melanoma [13].

At least three prospective phase II studies are exploring the role of the checkpoint inhibitors pembrolizumab and nivolumab in metastatic STS/bone sarcomas and/or uterine LMS; preliminary data were recently presented at the ASCO 2016 conference for two studies. Pembrolizumab showed some interesting responses in undifferentiated pleomorphic sarcoma (4/9), liposarcoma (2/9), synovial (1/9), chondrosarcoma (1/6) and osteosarcoma (1/19); no responses were seen in LMS (0/10) and Ewing sarcoma $(1 / 13)$ [14]. Interestingly in our series, we also observed a partial response in one patient with a dedifferentiated chondrosarcoma and a PD-L1 expression that was higher compared to all other tested patients (20\% versus less than 5\%); additionally, Kostine et al. [15] recently showed that this specific subtype of bone sarcoma expresses PD-L1 in association with immune-infiltrating cells and HLA class I in nearly $50 \%$ of cells. Immunotherapy with check-point inhibitors seems a particularly promising approach for the treatment of this rare and challenging histology but more data is needed.

A second prospective study is exploring nivolumab in 12 patients with uterine LMS and showed no responses [16]. In our series, among seven patients with LMS we observed 4 PD and 3 SD. LMS is characterized by a significant degree of morphologic and molecular heterogeneity and different molecular subtypes may respond differently to immunotherapy [17].

The combination of nivolumab and pazopanib is interesting but needs dose optimization to prevent, in particular, excessive liver toxicity. Nivolumab at $2 \mathrm{mg} / \mathrm{kg}$ every 3 weeks has been combined with pazopanib at $800 \mathrm{mg}$ po daily in patients with renal cell carcinoma; about $70 \%$ of patients experienced grade 3-4 side effects, mainly LFT abnormalities, fatigue and diarrhea [18].

\section{Conclusion}

We describe a cohort of 28 sarcoma patients with metastatic or unresectable soft tissue or bone sarcomas who were treated with the anti-PD-1 antibody nivolumab with or without the tyrosine kinase inhibitor pazopanib; we found evidence of clinical benefit with a half of the evaluable patients experiencing a partial response or a stabilization of disease after at least 4 cycles of nivolumab. Given the potential activity of nivolumab alone and promising data when combined with pazopanib, we are planning a prospective, phase II randomized study of nivolumab alone versus nivolumab with pazopanib, in metastatic soft tissue and bone sarcomas; correlative studies will include tumor and serum sampling for correlation with the clinical endpoints of response and progression-free survival.

\section{Additional file}

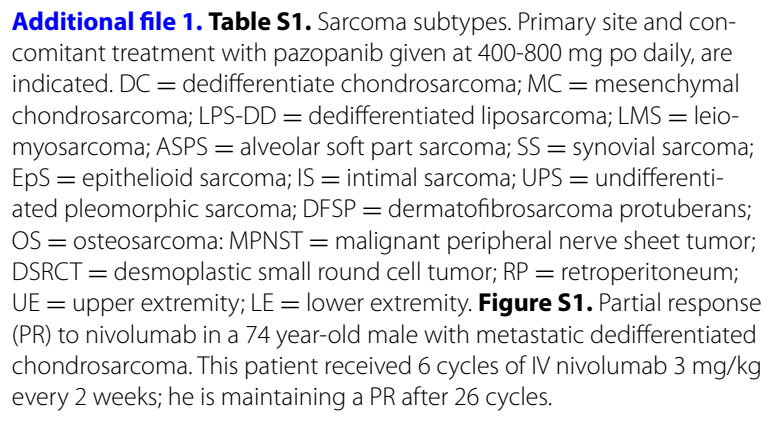

Additional file 1. Table S1. Sarcoma subtypes. Primary site and concomitant treatment with pazopanib given at 400-800 mg po daily, are indicated. $\mathrm{DC}=$ dedifferentiate chondrosarcoma; $\mathrm{MC}=$ mesenchymal chondrosarcoma; LPS-DD = dedifferentiated liposarcoma; LMS = leiomyosarcoma; ASPS = alveolar soft part sarcoma; SS = synovial sarcoma; EpS = epithelioid sarcoma; IS = intimal sarcoma; UPS = undifferentiated pleomorphic sarcoma; DFSP = dermatofibrosarcoma protuberans; OS = osteosarcoma: MPNST = malignant peripheral nerve sheet tumor; $\mathrm{DSRCT}=$ desmoplastic small round cell tumor; RP = retroperitoneum; $U E=$ upper extremity; LE = lower extremity. Figure S1. Partial response (PR) to nivolumab in a 74 year-old male with metastatic dedifferentiated chondrosarcoma. This patient received 6 cycles of IV nivolumab 3 mg/kg every 2 weeks; he is maintaining a PR after 26 cycles.

\section{Abbreviations}

pts: patients; STS: soft tissue sarcoma; PD-1: programmed death 1; PDL-1: programmed death ligand 1; PCR: polymerase chain reaction; TILs: tumor infiltrating lymphocytes; LFT: liver function tests; AST: aspartate transaminase; ALT: alanine transaminase; LMS: leiomyosarcoma; OS: osteosarcoma; DC: dedifferentiated chondrosarcoma; EpS: epithelioid sarcoma; IS: intimal sarcoma; SS: synovial sarcoma; MPNST: malignant peripheral nerve sheet tumor; MC: 
mesenchymal chondrosarcoma; LPS: liposarcoma; UPS: undifferentiated pleomorphic sarcoma; DSRCT: desmoplastic small round cell tumor; RMS: rhabdomyosarcoma; ASPS: alveolar soft part sarcoma; AE: adverse event; PD: progression of disease; SD: stable disease; PR: partial response; PFS: progression free survival; CTLs: cytotoxic T-lymphocytes.

\section{Authors' contributions}

LP and GR conceived the study and the design. LP and AC carried out data collection. LP and JW drafted the manuscript. AR performed pathologic review and immunohistochemical analysis. MG and AK interpreted the radiographic data. All authors read and approved the final manuscript.

\section{Author details \\ ${ }^{1}$ Department of Medicine, NYU Langone Medical Center, New York, NY, USA. \\ 2 Department of Radiology, NYU Langone Medical Center, New York, NY, USA \\ ${ }^{3}$ Department of Pathology, New York University School of Medicine, Laura and Isaac Perlmutter Cancer Center, 10th floor, Room 1041, 160 East 34th street, New York, NY, USA.}

\section{Acknowledgements}

We thank Bristol-Myers Squibb for providing nivolumab under a patient assistance program.

\section{Competing interests}

Dr. Jeffrey Weber accepts honoraria for advisory boards, and consulting fees. He is named on a patent filed by Moffitt Cancer center for an Ipilimumab biomarker. All remaining authors declared that they have no competing interests.

\section{Availability of data and materials}

The data that support the findings of this study are available from New York University but restrictions apply to the availability of these data, which were used under license for the current study, and so are not publicly available. Data are however available from the authors upon reasonable request and with permission from the IRB of New York University.

\section{Consent for publication}

Written informed consent for publication of patient clinical details and images was obtained from the patients included in the figures.

\section{Ethic approval and consent to participate}

This study was approved by the NYU Institutional Review Board (IRB).

Received: 27 September 2016 Accepted: 26 November 2016 Published online: 30 December 2016

\section{References}

1. Siegel RL, Miller KD, Jemal A. Cancer statistics, 2016. Cancer J Clin. 2016;66(1):7-30.

2. Jo VY, Fletcher CD. WHO classification of soft tissue tumors: an update based on the 2013 (4th) edition. Pathology. 2014;46(2):95-104.

3. Pang A, Carbini M, Maki RG. Contemporary therapy for advanced softtissue sarcomas in adults: a review. JAMA Oncol. 2016;2(7):941-47.

4. Choudhury N, Nakamura Y. The importance of immunopharmacogenomics in cancer treatment: patient selection and monitoring for immune checkpoint antibodies. Cancer Sci. 2016;107(2):107-15.

5. D'Angelo SP, Shoushtari AN, Agaram NP, Kuk D, Qin LX, Carvajal RD, Dickson MA, Gounder M, Keohan ML, Schwartz GK, Tap WD. Prevalence of tumor-infiltrating lymphocytes and PD-L1 expression in the soft tissue sarcoma microenvironment. Hum Pathol. 2015;46(3):357-65.
6. Kim JR, Moon YJ, Kwon KS, Bae JS, Wagle S, Kim KM, Park HS, Lee H, Moon WS, Chung MJ, Kang MJ, Jang KY. Tumor infiltrating PD1-positive lymphocytes and the expression of PD-L1 predict poor prognosis of soft tissue sarcomas. PLoS ONE. 2013;8(12):e82870.

7. Griffioen AW, Mans LA, de Graaf AM, Nowak-Sliwinska P, de Hoog CL, de Jong TA, Vyth-Dreese FA, van Beijnum JR, Bex A, Jonasch E. Rapid angiogenesis onset after discontinuation of sunitinib treatment of renal cell carcinoma patients. Clin Cancer Res. 2012;18(14):3961-71.

8. van der Graaf WT, Blay JY, Chawla SP, Kim DW, Bui-Nguyen B, Casali PG, Schöffski P, Aglietta M, Staddon AP, Beppu Y, Le Cesne A, Gelderblom H, Judson IR, Araki N, Ouali M, Marreaud S, Hodge R, Dewji MR, Coens C, Demetri GD, Fletcher CD, Dei Tos AP, Hohenberger P, EORTC Soft Tissue and Bone Sarcoma Group, PALETTE study group. Pazopanib for metastatic soft-tissue sarcoma (PALETTE): a randomised, double-blind, placebo-controlled phase 3 trial. Lancet. 2012;379(9829):1879-86.

9. Lim J, Poulin NM, Nielsen TO. New strategies in sarcoma: linking genomic and immunotherapy approaches to molecular subtype. Clin Cancer Res. 2015;21(21):4753-9.

10. Barretina J, Taylor BS, Banerji S, Ramos AH, Lagos-Quintana M, Decarolis PL, Shah K, Socci ND, Weir BA, Ho A, Chiang DY, Reva B, Mermel CH, Getz G, Antipin Y, Beroukhim R, Major JE, Hatton C, Nicoletti R, Hanna M, Sharpe T, Fennell TJ, Cibulskis K, Onofrio RC, Saito T, Shukla N, Lau C, Nelander S, Silver SJ, Sougnez C, Viale A, Winckler W, Maki RG, Garraway LA, Lash A, Greulich H, Root DE, Sellers WR, Schwartz GK, Antonescu CR, Lander ES, Varmus HE, Ladanyi M, Sander C, Meyerson M, Singer S. Subtype-specific genomic alterations define new targets for soft-tissue sarcoma therapy. Nat Genet. 2010;42(8):715-21.

11. Tseng WW, Malu S, Zhang M, Chen J, Sim GC, Wei W, Ingram D, Somaiah N, Lev DC, Pollock RE, Lizée G, Radvanyi L, Hwu P. Analysis of the intratumoral adaptive immune response in well differentiated and dedifferentiated retroperitoneal liposarcoma. Sarcoma. 2015;2015:547460.

12. Burgess M, Tawbi H. Immunotherapeutic approaches to sarcoma. Curr Treat Options Oncol. 2015;16(6):26.

13 Chandra RA, Wilhite TJ, Balboni TA, Alexander BM, Spektor A, Ott PA, $\mathrm{Ng}$ AK, Hodi FS, Schoenfeld JD. A systematic evaluation of abscopal responses following radiotherapy in patients with metastatic melanoma treated with ipilimumab. Oncoimmunology. 2015;4(11):1046028.

14 Tawbi HA-H, Burgess MA, Crowley J, van Tine BA, Hu J, Schuetze S, D’Angelo SP, Attia S, Priebat DA, Okuno SH, Riedel RF, Davis LE, Movva S, Reed DR, Baker LH, Reinke DK, Maki RG, Patel S, for SARC028 Investigators. Safety and efficacy of PD-1 blockage using pembrolizumab in patients with advanced soft tissue (STS) and bone sarcomas (BS): results of SARC028-a multicenter phase II study. J Clin Onc. 2016;34(15):11006.

15 Kostine M, Cleven AH, de Miranda NF, Italiano A, Cleton-Jansen AM, Bovée JV. Analysis of PD-L1, T-cell infiltrate and HLA expression in chondrosarcoma indicates potential for response to immunotherapy specifically in the dedifferentiated subtype. Mod Pathol. 2016;29(9):1028-37.

16 George S, Barysauskas CM, Solomon S, Tahlil K, Malley R, Hohos M, Polson K, Loucks M, Wagner AJ, Merriam P, Morgan JA, Rodig SJ, Hodi FS, Shapiro $G$, Demetri GD. Phase II study of nivolumab in metastatic leiomyosarcoma of the uterus. J Clin Oncol. 34, 2016 (supplemental abstract 11007).

17 Beck AH, Lee CH, Witten DM, Gleason BC, Edris B, Espinosa I, Zhu S, Li R, Montgomery KD, Marinelli RJ, Tibshirani R, Hastie T, Jablons DM, Rubin $B P$, Fletcher CD, West RB, van de Rijn M. Discovery of molecular subtypes in leiomyosarcoma through integrative molecular profiling. Oncogene. 2010;29(6):845-54.

18 Amin A, Plimack ER, Infante JR, Ernstoff MS, Rini BI, McDermott DF, Knox JJ, Pal SK, Voss MH, Sharma P, Kollmannsberger CK, Chin Heng DY, Spratlin $J$ L, Shen Y, Kurland JF, Gagnier P, Hammer HJ. Nivolumab (anti-PD-1; BMS-936558, ONO-4538) in combination with sunitinib or pazopanib in patients (pts) with metastatic renal cell carcinoma (mRCC). ASCO. J Clin Oncol. 2014;32(5) (supplemental abstract 5010) 